International Journal of Pure and Applied Mathematics

Volume 115 No. 2 2017, 369-382

ISSN: 1311-8080 (printed version); ISSN: 1314-3395 (on-line version)

url: http://www.ijpam.eu

doi: 10.12732 /jpam.v115i2.14

\title{
GENERALIZED FIXED POINT THEOREMS IN G-METRIC SPACE UNDER AN IMPLICIT RELATION
}

T. Phaneendra

Department of Mathematics

School of Advanced Sciences

VIT University

Vellore, 632014, Tamil Nadu, INDIA

\begin{abstract}
Common fixed point theorems are proved in a G-metric space for four self-maps through the notions of weak compatibility, common limit range property and an implicit relation. These are generalized versions of the results of [3], [4] and the metrical fixed point theorem obtained in [5].
\end{abstract}

AMS Subject Classification: $54 \mathrm{H} 25$

Key Words: G-metric space, common limit range property, implicit relation, weakly compatible maps, coincidence point, common fixed point

\section{Introduction}

Let $X$ be a nonempty set and $G: X \times X \times X \rightarrow \mathbb{R}$ such that

(G1) $G(x, y, z) \geq 0$ for all $x, y, z \in X$ with $G(x, y, z)=0$ if $x=y=z$,

(G2) $G(x, x, y)>0$ for all $x, y \in X$ with $x \neq y$,

(G3) $G(x, x, y) \leq G(x, y, z)$ for all $x, y, z \in X$ with $z \neq y$,

(G4) $G(x, y, z)=G(x, z, y)=G(y, x, z)=G(z, x, y)$

$=G(y, z, x)=G(z, y, x)$ for all $x, y, z \in X$

(G5) $G(x, y, z) \leq G(x, w, w)+G(w, y, z)$ for all $x, y, z, w \in X$

Received: February 23, 2017

Revised: $\quad$ May 23, 2017

Published:

July 14, 2017
(C) 2017 Academic Publications, Ltd. url: www.acadpubl.eu 
Then $G$ is called a $G$-metric on $X$ and the pair $(X, G)$ denotes a $G$-metric space. Axiom (G5) is known as the rectangle inequality (of $G$ ). This notion was introduced by Mustafa and Sims [1] as a generalization of metric space. From this definition, it immediately follows that

$$
G(x, y, y)=0 \quad \Rightarrow \quad x=y \quad \text { for all } \quad x, y \in X
$$

and

$$
G(x, y, y) \leq 2 G(x, x, y) \text { for all } x, y \in X .
$$

A $G$-metric space $(X, G)$ is said to be symmetric, if $G(x, y, y)=G(y, x, x)$ for all $x, y \in X$.

Example 1.1. Let $(X, d)$ be a metric space. Define

$$
G(x, y, z)=d(x, y)+d(y, z)+d(z, x) \text { for all } x, y, z \in X
$$

Then $(X, G)$ is a symmetric $G$-metric space.

Let $x, y$ and $z$ be the vertices of a triangle in a plane. Then $d(x, y)$ denotes the length of the side joining $x$ and $y$ and $G(x, y, z)$ represents the perimeter of the triangle.

The following terminology was developed by Mustafa et al in [1] and [2]:

Definition 1.1. A sequence $\left\langle x_{n}\right\rangle_{n=1}^{\infty} \subset X$ is said to be $G$-convergent with limit $p \in X$, if $\lim _{n, m \rightarrow \infty} G\left(p, x_{n}, x_{m}\right)=0$, that is, if for any $\epsilon>0$ there is a positive integer $N$ such that $G\left(x_{n}, x_{m}, p\right)<\epsilon$ for all $n, m \geq N$.

Lemma 1.1. The following statements are equivalent in a $G$-metric space $(X, G)$ :

(a) $\left\langle x_{n}\right\rangle_{n=1}^{\infty} \subset X$ is G-convergent with limit $p \in X$,

(b) $\lim _{n \rightarrow \infty} G\left(x_{n}, x_{n}, p\right)=0$,

(c) $\lim _{n \rightarrow \infty} G\left(x_{n}, p, p\right)=0$.

First we give the following useful notation in a $G$-metric space $(X, G)$ :

Definition 1.2. A point $p \in X$ is a coincidence point of self-maps $f$ and $r$ on $X$, if $f p=r p=u$, where $u$ is a point of coincidence of $f$ and $r$.

Definition 1.3. Self-maps $f$ and $r$ on $X$ are weakly compatible, if they commute at their coincidence point. 
Definition 1.4. Self-maps $f$ and $r$ on $X$ satisfy the property $(E A)$, if there is a sequence $\left\langle x_{n}\right\rangle_{n=1}^{\infty}$ in $X$ such that

$$
\lim _{n \rightarrow \infty} f x_{n}=\lim _{n \rightarrow \infty} r x_{n}=u \text { for some } u \in X .
$$

Definition 1.5. Self-maps $f$ and $r$ on $X$ satisfy the $C L R_{r}$-property if there is a sequence $\left\langle x_{n}\right\rangle{ }_{n=1}^{\infty}$ in $X$ such that

$$
\lim _{n \rightarrow \infty} f x_{n}=\lim _{n \rightarrow \infty} r x_{n}=r p \quad \text { for some } \quad p \in X .
$$

With these ideas, the following was proved in [3]:

Theorem 1.1. Let $f$ and $r$ be self-maps on $X$ such that

$$
\begin{array}{r}
G(f x, f y, f z) \leq k \max \{G(r x, r y, r z), G(r x, f x, f x), G(r x, f y, f y), \\
G(r x, f z, f z),, G(r y, f y, f y)), G(r y, f x, f x), \\
G(r y, f z, f z), G(r z, f z, f z), G(r z, f x, f x), \\
G(r z, f y, f y)\} \text { for all } x, y, z \in X,
\end{array}
$$

where $0 \leq k<1 / 4$. If $(f, r)$ satisfies the $C L R_{r}$-property and $r$ is weakly compatible with $f$, then $f$ and $r$ will have a unique common fixed point.

Writing $z=y$, we immediately get

Corollary 1.1. Let $f$ and $r$ be self-maps on $X$ such that

$$
\begin{array}{r}
G(f x, f y, f y) \leq k \max \{G(r x, r y, r y), G(r x, f x, f x), G(r y, f y, f y), \\
G(r x, f y, f y), G(r y, f x, f x)\} \text { for all } x, y \in X,
\end{array}
$$

where $0 \leq k<1 / 4$. If $(f, r)$ satisfies the $C L R_{r}$-property and $r$ is weakly compatible with $f$, then $f$ and $r$ will have a unique common fixed point.

Theorem 1.2 (Theorem 2.5, [4]). Let $f$ and $r$ be self-maps on $X$ and for all $x, y \in X$ either

$$
\begin{aligned}
G(f x, f y, f y) \leq q \max \{ & G(r y, f y, f y), \\
& G(r x, f y, f y), G(r y, f x, f x)\}
\end{aligned}
$$

or

$$
\begin{aligned}
G(f x, f y, f y) \leq q \max \{ & G(r y, r y, f y), \\
& G(r x, r x, f y), G(r y, r y, f x)\}
\end{aligned}
$$

holds good, where $0 \leq q<1$. If the range of $f$ is contained in the range of $r$ and $r(X)$ is a complete subspace of $X$, then $f$ and $r$ will have a unique common fixed point, provided $r$ is weakly compatible with $f$. 
Remark 1.1. When the range of values of $q$ is restricted to $[0,1 / 4)$, the right hand side of (1.8) is less than or equal to the right hand side of (1.7). In other words, (1.7) will be weaker than (1.8), when $q \in[0,1 / 4)$. Also, given $x_{0} \in X$, if $f(X) \subset r(X)$, then we can define the sequence $\left\langle x_{n}\right\rangle{ }_{n=1}^{\infty}$ in $X$ with the choice

$$
f x_{n-1}=r x_{n} \text { for } n=1,2,3, \ldots
$$

It can be shown that $\left\langle r x_{n}\right\rangle_{n=1}^{\infty}$ is a Cauchy sequence in $r(X)$ and hence converges in it, provided $r(X)$ is complete. Thus $(f, r)$ satisfies the $C L R_{r}$-property whenever $f(X) \subset r(X)$ and $r(X)$ is complete. Hence Corollary 1.1 is a partial generalization of Theorem 1.2 when $q \in[0,1 / 4)$.

In this paper, first we extend Corollary 1.1 to four self-maps using the notion of an implicit relation ( $c f$. Section 2), which is a generalization of the metrical fixed point theorem proved in [5]. Then we derive a generalization of Theorem 1.2 under a certain condition by slightly altering the contraction conditions of the first result.

\section{Main Results and Discussion}

The notion of implicit-type relations were first introduced by Popa [6] to cover several contractive conditions and unify fixed point theorems in metric spaces (See [7], [8], [9] and so on). Recently Popa and Patriciu [10] inserted a continuous implicit relation $\phi: \mathbb{R}_{+}^{4} \rightarrow \mathbb{R}$ to prove fixed point theorems in a $G$-metric spaces. In this paper, we employ a lower semicontinuous implicit function $\psi: \mathbb{R}_{+}^{6} \rightarrow \mathbb{R}$, which is nondecreasing in each coordinate variable, such that

$\left(P_{a}\right) \psi(l, 0,0, l, l, 0)>0$ for all $l>0$,

$\left(P_{b}\right) \psi(l, 0, l, 0,0, l)>0$ for all $l>0$,

$\left(P_{c}\right) \psi(l, l, 0,0, l, l)>0$ for all $l>0$.

Example 2.1. Let $\psi\left(t_{1}, t_{2}, t_{3}, t_{4}, t_{5}, t_{6}\right)=t_{1}-\max \left\{t_{2}, \frac{t_{3}+t_{4}}{2}, \frac{t_{5}+t_{6}}{2}\right\}$.

Example 2.2. Let $\psi\left(t_{1}, t_{2}, t_{3}, t_{4}, t_{5}, t_{6}\right)=t_{1}-\max \left\{t_{2}, \beta t_{3}+\alpha t_{4} \frac{t_{5}+t_{6}}{2}\right\}$, where $\beta \geq 0$ and $0<\alpha<1$.

Example 2.3. Let $\psi\left(t_{1}, t_{2}, t_{3}, t_{4}, t_{5}, t_{6}\right)=t_{1}-\max \left\{t_{2}, \alpha t_{3}, \alpha t_{4}, \frac{t_{5}+t_{6}}{2}\right\}$, where $0<\alpha<1$.

Example 2.4. Let $\psi\left(t_{1}, t_{2}, t_{3}, t_{4}, t_{5}, t_{6}\right)=t_{1}-\max \left\{t_{2}, \frac{t_{3}+t_{4}}{2}, \frac{t_{5}+t_{6}}{2}\right\}$. 
Example 2.5. Let $\psi\left(t_{1}, t_{2}, t_{3}, t_{4}, t_{5}, t_{6}\right)=t_{1}-\left[a t_{2}+b t_{3}+c t_{4}+e\left(t_{5}+t_{6}\right)\right]$, where $a, b, c$ and $e$ are nonnegative numbers with $a+b+c+2 e<1$.

Example 2.6. Let $\psi\left(t_{1}, t_{2}, t_{3}, t_{4}, t_{5}, t_{6}\right)=t_{1}^{3}-\left[a t_{1}^{2} t_{2}+b t_{1} t_{3} t_{4}+c t_{5}^{2} t_{6}+d t_{5} t_{6}^{2}\right]$, where $a>0, b, c, e \geq 0$ such that $a+c+e<1$ and $a+b<1$.

Example 2.7. Let $\psi\left(l_{1}, l_{2}, l_{3}, l_{4}, l_{5}, l_{6}\right)=l_{1}^{2}-a l_{2}^{2}-\frac{b l_{5} l_{6}}{l_{3}^{2}+l_{4}^{2}+1}$, where $a=1 / 2$ and $b=1 / 4$.

Our first main result is

Theorem 2.1. Let $f, g, h$ and $r$ be self-maps on $X$ such that for all $x, y \in X$ any two of the following inequalities hold good:

$$
\begin{gathered}
\psi(G(f x, g y, g y), G(r x, r y, r y), G(r x, f x, f x), G(r y, g y, g y), \\
G(r x, g y, g y), G(r y, f x, f x)) \leq 0, \\
\psi(G(g x, h y, h y), G(r x, r y, r y), G(r x, g x, g x), G(r y, h y, h y), \\
G(r x, h y, h y), G(r y, g x, g x)) \leq 0, \\
\psi(G(h x, f y, f y), G(r x, r y, r y), G(r x, h x, h x), G(r y, f y, f y), \\
G(r x, f y, f y), G(r y, h x, h x)) \leq 0 .
\end{gathered}
$$

Suppose that one of the pairs $(f, r),(g, r)$ and $(h, r)$ satisfies the $C L R_{r}$-property. If $r$ is weakly compatible with any one of $f, g$ and $h$, then all the four maps $f$, $g, h$ and $r$ will have a common coincidence point $u$, which will be their unique common fixed point.

Proof. Suppose $f$ and $r$ satisy the $C L R_{r}$-property. Then we can find a $\left\langle x_{n}\right\rangle{ }_{n=1}^{\infty} \subset X$ such that (1.5) holds good.

First we prove that

$$
\lim _{n \rightarrow \infty} f x_{n}=\lim _{n \rightarrow \infty} g x_{n}=\lim _{n \rightarrow \infty} h x_{n}=\lim _{n \rightarrow \infty} r x_{n}=r p .
$$

Writing $x=y=x_{n}$ in $(2.1)$, we get

$$
\begin{aligned}
& \psi\left(G\left(f x_{n}, g x_{n}, g x_{n}\right), G\left(r x_{n}, r x_{n}, r x_{n}\right), G\left(r x_{n}, f x_{n}, f x_{n}\right)\right. \\
& \left.\quad G\left(r x_{n}, g x_{n}, g x_{n}\right), G\left(r x_{n}, g x_{n}, g x_{n}\right), G\left(r x_{n}, f x_{n}, f x_{n}\right)\right) \leq 0 .
\end{aligned}
$$

Applying the limit as $n \rightarrow \infty$ and then using (1.5) and the lower semicontinuity of $\psi$, this yields

$$
\psi(G(r p, q, q), 0,0, G(r p, q, q), G(r p, q, q), 0) \leq 0
$$


where $q=\lim _{n \rightarrow \infty} g x_{n}$. If $G(r p, q, q)>0$, then $(2.5)$ gives a contradiction to $\left(P_{a}\right)$. Thus $G(r p, q, q)=0$ so that $q=r p$, in view of (1.1).

Again, taking $x=y=x_{n}$ in (2.2), we get

$$
\begin{gathered}
\psi\left(G\left(g x_{n}, h x_{n}, h x_{n}\right), G\left(r x_{n}, r x_{n}, r x_{n}\right), G\left(r x_{n}, g x_{n}, g x_{n}\right), G\left(r x_{n}, h x_{n}, h x_{n}\right),\right. \\
\left.G\left(r x_{n}, h x_{n}, h x_{n}\right), G\left(r x_{n}, g x_{n}, g x_{n}\right)\right) \leq 0,
\end{gathered}
$$

Applying the limit as $n \rightarrow \infty$ and the lower semicontinuity of $\psi$, this yields

$$
\psi(G(r p, t, t), 0,0, G(r p, t, t), G(r p, t, t), 0) \leq 0,
$$

where $\lim _{n \rightarrow \infty} h x_{n}=t$. This would also give a contradiction to $\left(P_{a}\right)$ if $G(r p, t, t)>$ 0 . Thus $G(r p, t, t)=0$ so that $t=r p$, in view of (1.1). This proves (2.4).

Similarly (2.4) can be established whenever $(g, r)$ or $(h, r)$ satisfies the $C L R_{r}$-property under (2.1) and (2.2). The other cases that one of $(f, r),(g, r)$ and $(h, r)$ satisfies the $C L R_{r}$-property under [(2.2) and (2.3)] or [(2.1) and (2.3)] will prove (2.4).

We shall prove that $f, g, h$ and $t$ have a common coincidence in the following three cases:

Case (a): $(f, r)$ is weakly compatible,

Case (b): $(g, r)$ is weakly compatible,

Case (c): $(h, r)$ is weakly compatible

Case (a) $(f, r)$ is weakly compatible and any one of the pairs [(2.1), (2.2)], $[(2.1),(2.3)]$ and $[(2.2),(2.3)]$ holds good:

$$
f p=r p .
$$

If possible we assume that $f p \neq r p$ so that $l=G(r p, f p, f p)>0$ and $k=$ $G(f p, f p, r p)>0$ by (G4), and $l \geq k / 2$, by (1.2).

Then writing $x=p$ and $y=x_{n}$ in (2.1), we get

$$
\begin{aligned}
& \psi\left(G\left(f p, g x_{n}, g x_{n}\right), G\left(r p, r x_{n}, r x_{n}\right), G(r p, f p, f p),\right. \\
& \left.\quad G\left(r x_{n}, g x_{n}, g x_{n}\right), G\left(r p, g x_{n}, g x_{n}\right), G\left(r x_{n}, f p, f p\right)\right) \leq 0 .
\end{aligned}
$$

Applying the limit as $n \rightarrow \infty$ and using (2.4) and the lower semicontinuity of $\psi$, this yields

$$
\psi(k, 0, l, 0,0, l) \leq 0
$$


Since $\psi$ is nondecreasing in each coordinate variable, the above imlpies that

$$
\psi\left(\frac{k}{2}, 0, \frac{k}{2}, 0,0, \frac{k}{2}\right) \leq \psi(k, 0, l, 0,0, l) \leq 0
$$

which contradicts the choice $\left(P_{b}\right)$. Therefore (2.6) must hold good.

Since $f$ and $r$ commute at the coincidence point $p$, it follows that $f r p=r f p$ or

$$
f u=r u, \text { where } f p=r p=u \text {. }
$$

Again, (2.1) with $x=y=u$ and (2.7) gives

$$
\begin{aligned}
& \psi(G(f u, g u, g u), G(r u, r u, r u), G(r u, f u, f u) \\
& \quad G(r u, g u, g u), G(r u, g u, g u), G(r u, f u, f u)) \leq 0
\end{aligned}
$$

or

$$
\psi(G(f u, g u, g u), 0,0, G(f u, g u, g u), G(f u, g u, g u), 0) \leq 0,
$$

which will contradict with $\left(P_{a}\right)$ if $G(f u, g u, g u)>0$.

Hence $G(f u, g u, g u)=0$ so that (1.1) gives $f u=g u=r u$.

Suppose that (2.2) holds good. With $x=u=y$, this gives

$$
\begin{aligned}
& \psi(G(g u, h u, h u), G(r u, r u, r u), G(r u, g u, g u) \\
& G(r u, h u, h u), G(r u, h u, h u), G(r u, g u, g u)) \leq 0
\end{aligned}
$$

or that $\psi(G(g u, h u, h u), 0,0, G(g u, h u, h u), G(g u, h u, h u), 0) \leq 0$.

This again contradicts with $\left(P_{a}\right)$ if $G(g u, h u, h u)>0$ so that

$$
G(g u, h u, h u)=0 \text { or } g u=h u \text {. }
$$

In other words, $u$ is a common coincidence point of $f, g, h$ and $r$, that is

$$
f u=g u=h u=r u, \text { where } f p=r p=u \text {. }
$$

On the other hand, if (2.3) holds good, then writing $x=y=u$ in this, followed by (2.7) and $f u=g u$, and proceeding as above, we get $g u=h u$ and hence $(2.8)$.

In this way, (2.8) follows in either case [(2.1), (2.2)] and [(2.1), (2.3)].

Now consider the inequalities (2.2) and (2.3). 
Writing $x=x_{n}$ and $y=p$ in (2.3), we get

$$
\begin{aligned}
& \psi\left(G\left(h x_{n}, f p, f p\right), G\left(r x_{n}, r p, r p\right), G\left(r x_{n}, h x_{n}, h x_{n}\right),\right. \\
& \left.G(r p, f p, f p), G\left(r x_{n}, f p, f p\right), G\left(r p, h x_{n}, h x_{n}\right)\right) \leq 0 .
\end{aligned}
$$

Applying the limit as $n \rightarrow \infty$ and using (2.4) and the lower semicontinuity of $\psi$, we get

$$
\psi(G(r p, f p, f p), 0,0, G(r p, f p, f p), G(r p, f p, f p), 0) \leq 0 .
$$

This gives a contradiction to $\left(P_{a}\right)$ if $G(r p, f p, f p)>0$. Thus

$$
G(r p, f p, f p)=0 \text { or } r p=f p=u \text {, by }(1.1)
$$

and hence (2.7) follows from the weak compatibility of $(f, r)$. Again from (2.3) with $x=u=y$ and (2.7), we see that

$$
\begin{aligned}
& \psi(G(h u, f u, f u), G(r u, r u, r u), G(r u, h u, h u), \\
& \qquad(r u, f u, f u), G(r u, f u, f u), G(r u, h u, h u)) \leq 0
\end{aligned}
$$

or $\psi(G(h u, f u, f u), 0, G(f u, h u, h u), 0,0, G(f u, h u, h u)) \leq 0$. This, in view of (1.2) and the nondecreasing nature of $\psi$, gives

$$
\psi\left(\frac{G(h u, f u, f u)}{2}, 0, \frac{G(h u, f u, f u)}{2}, 0,0, \frac{G(h u, f u, f u)}{2}\right) \leq 0 .
$$

This would be against $\left(P_{b}\right)$ if $G(f u, f u, h u)>0$. Therefore, $G(f u, f u, h u)=0$ or $f u=h u$ and hence $f u=h u=r u$.

But then, (2.2) with $x=u=y$ and (2.7) imply that

$$
\psi(G(g u, f u, f u), 0, G(f u, g u, g u), 0,0, G(f u, g u, g u)) \leq 0,
$$

This also, in view of (1.2) and the nondecreasing nature of $\psi$, gives

$$
\psi\left(\frac{G(g u, f u, f u)}{2}, 0, \frac{G(g u, f u, f u)}{2}, 0,0, \frac{G(g u, f u, f u)}{2}\right) \leq 0,
$$

which again contradicts $\left(P_{b}\right)$ if $G(f u, f u, g u)>0$. Thus $G(f u, f u, g u)=0$ so that $f u=g u$, from (1.1), and thus (2.8) follows.

Case (b): Let $(g, r)$ be weakly compatible.

Subcase (i): Apply (2.1) with $x=x_{n}$ and $y=p$ to get $p$ as a coincidence point of $g$ and $r$ and hence to get $u=g p=r p$ as their coincidence point. Then 
we again use (2.1) with $x=y=u$ to get $u$ as a coincidence point of $f$ and $g$. Further we use (2.2) with $x=y=u$ so that $u$ will be a coincidence point of $g$ and $h$. Or else we use (2.3) with $x=y=u$ to get $u$ as a coincidence point of $f$ and $h$.

Subcase (ii): Apply (2.2) with $x=p$ and $y=x_{n}$ to get $p$ as a coincidence point of $g$ and $r$ and hence to get $v=g p=r p$ as their coincidence point. Then we again use (2.2) with $x=y=v$ to get $v$ as a coincidence point of $g$ and $h$. Further we use (2.3) with $x=y=v$ so that $v$ will be a coincidence point of $h$ and $f$. Or else we use (2.1) with $x=y=v$ to get $v$ as a coincidence point of $f$ and $g$.

Case (c): Let $(h, r)$ be weakly compatible.

Subcase (i): Apply (2.2) with $x=x_{n}$ and $y=p$ to get $p$ as a coincidence point of $h$ and $r$, and to get $w=h p=r p$ also as their coincidence point. Then we again use (2.2) with $x=y=w$ to get $w$ as a coincidence point of $g$ and $h$. Further we use (2.3) with $x=y=w$ so that $w$ will be a coincidence point of $h$ and $f$. Or else we use (2.1) with $x=y=w$ to get $w$ as a coincidence point of $f$ and $g$.

Subcase (ii): Apply (2.3) with $x=p$ and $y=x_{n}$ to get $p$ as a coincidence point of $h$ and $r$, and hence $z=h p=r p$ also as their coincidence point. Then we again use (2.3) with $x=y=z$ to get $z$ as a coincidence point of $h$ and $f$. Further we use (2.1) with $x=y=z$ so that $z$ is a coincidence point of $f$ and $g$.

Thus we get (2.8) in all the cases.

Now we employ (2.1) with $x=u$ and $y=x_{n}$, which in the limit as $n \rightarrow \infty$ gives $u$ as a fixed point of $f$ and hence a common fixed point of $f, g, h$ and $r$. In fact, (2.1) with $x=u$ and $y=x_{n}$ gives

$$
\begin{aligned}
& \psi\left(G\left(f u, g x_{n}, g x_{n}\right), G\left(r u, r x_{n}, r x_{n}\right), G(r u, f u, f u)\right. \\
& \left.\quad G\left(r x_{n}, g x_{n}, g x_{n}\right), G\left(r u, g x_{n}, g x_{n}\right), G\left(r x_{n}, f u, f u\right)\right) \leq 0 .
\end{aligned}
$$

Proceeding the limit as $n \rightarrow \infty$, in this and using (2.8) and lower semicontinuity of $\psi$, we obtain

$$
\psi(G(f u, u, u), G(f u, u, u), 0,0, G(f u, u, u), G(u, f u, f u)) \leq 0
$$

which from (1.2) gives

$$
\psi\left(\frac{G(f u, u, u)}{2}, \frac{G(f u, u, u)}{2}, 0,0, \frac{G(f u, u, u)}{2}, \frac{G(f u, u, u)}{2}\right) \leq 0,
$$


since $\psi$ is nondecreasing. This is a contradiction to $\left(P_{c}\right)$ if $G(f u, u, u)>0$, proving that $G(f u, u, u)=0$ or $f u=u$, in view of (1.1). This together with (2.8) implies that $u$ is a common fixed point of $f, g, h$ and $r$.

To establish the uniqueness of the common fixed point, we assume that $a$ and $b$ are two distinct common fixed points of $f, g, h$ and $r$ so that $G(a, b, b)>0$ and

$$
f a=g a=h a=r a=a \quad \text { and } \quad f b=g b=h b=r b=b .
$$

Then writing $x=a$ and $y=b$ in (2.1), we get

$$
\begin{aligned}
& \psi(G(f a, g b, g b), G(r a, r b, r b), G(r a, f a, f a), \\
& G(r b, g b, g b), G(r a, g b, g b), G(r b, f a, f a)) \leq 0 .
\end{aligned}
$$

Using (2.9) in this, we obtain

$$
\psi(G(a, b, b), G(a, b, b), 0,0, G(a, b, b), G(b, a, a)) \leq 0
$$

or

$$
\psi\left(\frac{G(a, b, b)}{2}, \frac{G(a, b, b)}{2}, 0,0, \frac{G(a, b, b)}{2}, \frac{G(a, b, b)}{2}\right) \leq 0
$$

which leads to a contradiction to $\left(P_{c}\right)$. Therefore $a=b$.

In other words, the common fixed point of $f, g, h$ and $r$ is unique.

Taking $g=h=f$ in Theorem 2.1, we get

Corollary 2.1. Let $f$ and $r$ be self-maps on $X$ such that

$$
\begin{array}{r}
\psi(G(f x, f y, f y), G(r x, r y, r y), G(r x, f x, f x), G(r y, f y, f y), \\
G(r x, f y, f y), G(r y, f x, f x)) \leq 0 \text { for all } x, y \in X,
\end{array}
$$

Suppose that $(f, r)$ satisfies the $C L R_{r}$-property. If $r$ is weakly compatible with $f$, then $f$ and $r$ will have a coincidence point $u$, which will be their unique common fixed point.

To obtain the result of [5] as an important consequence of Theorem 2.1, we need the following notions in a metric space $(X, d)$ :

Definition 2.1. Given $x_{0} \in X$ and $f, g, h$ and $r$ self-maps on $X$, if there exist points $x_{1}, x_{2}, x_{3}, \ldots$ in $X$ such that

$$
f x_{3 n-3}=r x_{3 n-2}, g x_{3 n-2}=r x_{3 n-1}, h x_{3 n-1}=r x_{3 n}, n=1,2,3, \ldots,
$$


then $\left\langle r x_{n}\right\rangle_{n=1}^{\infty}$ is an $(f, g, h)$-orbit at $x_{0}$ relative to $r$.

The space $X$ is $(f, g, h)$-orbitally complete at $x_{0}$ relative to $r$ if every Cauchy sequence in an $(f, g, h)$-orbit at $x_{0}$ relative to $r$ converges in $X$, and $X$ is $(f, g, h)$-orbitally complete relative to $r$ if it is $(f, g, h)$-orbitally complete at each $x_{0} \in X$ relative to $r$.

Definition 2.2. Self-maps $f, g, h$ and $r$ satisfy the property (EA) if there exists a sequence $\left\langle x_{n}\right\rangle_{n=1}^{\infty}$ in $X$ such that

$$
\lim _{n \rightarrow \infty} f x_{n}=\lim _{n \rightarrow \infty} g x_{n}=\lim _{n \rightarrow \infty} h x_{n}=\lim _{n \rightarrow \infty} r x_{n}=u \text { for some } u \in X .
$$

The following was the main result proved in [5]]:

Corollary 2.2. Let $f, g, h$ and $r$ be self-maps on a metric space $(X, d)$ satisfying the property $(E A)$. For all $x, y \in X$, suppose that any two of the following inequalities hold good:

$$
\begin{aligned}
& \psi(d(f x, g y), d(r x, r y), d(r x, f x), \\
& d(r y, g y), d(r x, g y), d(r y, f x)) \leq 0, \\
& \psi(d(g x, h y), d(r x, r y), d(r x, g x), \\
& d(r y, h y), d(r x, h y), d(r y, g x)) \leq 0, \\
& \psi(d(h x, f y), d(r x, r y), d(r x, h x), \\
& d(r y, f y), d(r x, f y), d(r y, h x)) \leq 0 .
\end{aligned}
$$

Suppose that $r(X)$ is $(f, g, h)$-orbitally complete relative to $r$ and $r$ is weakly compatible with any one of $f, g$ and $h$, then all the four maps $f, g, h$ and $r$ will have a unique common coincidence point which will also be their unique common fixed point.

Remark 2.1. Let $(X, d)$ be a metric space. Define the $G$ metric as in Example 1.1. Then $(X, G)$ is a symmetric $G$-metric space and

$$
\begin{aligned}
& G(f x, g y, g y)=d(f x, g y), G(r x, r y, r y)=d(r x, r y), \\
& G(r x, f x, f x)=d(r x, f x) \text { etc. for all } x, y \in X
\end{aligned}
$$

Hence (2.13)-(2.15) are particular cases of (2.1)-(2.3) respectively. Since the property (EA) and the orbital completeness of $r(X)$ imply the $C L R_{r}$-property, we see that the conclusion of Theorem 2.2 follows from that of Theorem 2.1.

Next we see that Corollary 1.1 is a particular case of Corollary 2.1. 
Remark 2.2. We write

$\psi\left(t_{1}, t_{2}, t_{3}, t_{4}, t_{5}, t_{6}\right)=t_{1}-k \max \left\{t_{2}, t_{3}, t_{4}, t_{5}, t_{6}\right\}$ for all $t_{i} \geq 0, i=1,2, \ldots, 6$,

where $0 \leq k<1 / 4$. Then (1.7) follows from (2.19) and hence Corollary 1.1 is a particular case of Corollary 2.1. In view of the choice of $k$, it may be noted from the proof of Corollary 1.1 given in [3], that the symmetry of $X$ can be dropped when the inequality (2.10) is condensed as (1.7) .

Slightly altering the inequalities (2.1)-(2.3), one can obtain the following result:

Theorem 2.2. Let $f, g, h$ and $r$ be self-maps on a symmetric $G$-metric space $(X, G)$ such that for all $x, y \in X$ any two of the following inequalities hold good:

$$
\begin{gathered}
\psi(G(f x, g y, g y), G(r x, r x, r y), G(r x, r x, f x), G(r y, r y, g y), \\
G(r x, r x, g y), G(r y, r y, f x)) \leq 0, \\
\psi(G(g x, h y, h y), G(r x, r x, r y), G(r x, r x, g x), G(r y, r y, h y), \\
G(r x, r x, h y), G(r y, r y, g x)) \leq 0, \\
\psi(G(h x, f y, f y), d(r x, r x, r y), G(r x, r x, h x), G(r y, r y, f y), \\
G(r x, r x, f y), G(r y, r y, h x)) \leq 0 .
\end{gathered}
$$

Suppose that one of the pairs $(f, r),(g, r)$ and $(h, r)$ satisfies the $C L R_{r}$-property. If $r$ is weakly compatible with any one of $f, g$ and $h$, then all the four maps $f$, $g, h$ and $r$ will have a common coincidence point $u$, which will be their unique common fixed point.

Again with $g=h=f$ in Theorem 2.2, we get

Corollary 2.3. Let $f$ and $r$ be self-maps on $X$ such that

$$
\begin{gathered}
\psi(G(f x, f y, f y), G(r x, r x, r y), G(r x, r x, f x), G(r y, r y, f y) \\
G(r x, r x, f y), G(r y, r y, f x)) \leq 0 \text { for all } x, y \in X
\end{gathered}
$$

If $(f, r)$ satisfies the $C L R_{r}$-property and $r$ is weakly compatible with $f$, then $f$ and $r$ will have a coincidence point $u$. Further if $X$ is symmetric, then $u$ will become their unique common fixed point.

Remark 2.3. The symmetry of $X$ is not used in Corollary 2.1 and Corollary 2.3 to obtain the coincidence point of $f$ and $r$, unlike in Theorem 2.2.

The following is a unification of Corollary 2.1 and Corollary 2.3, whose proof is simple: 
Corollary 2.4. Let $f$ and $r$ be self-maps on $X$ satisfying (2.10) or (2.19) for all $x, y \in X$. If $(f, r)$ satisfies the $C L R_{r}$-property and $r$ is weakly compatible with $f$, then $f$ and $r$ will have a coincidence point $u$. Further $u$ will become their unique common fixed point if $X$ is symmetric.

Remark 2.4. Again taking

$$
\psi\left(t_{1}, t_{2}, t_{3}, t_{4}, t_{5}, t_{6}\right)=t_{1}-q \max \left\{t_{4}, t_{5}, t_{6}\right\} \text { for all } t_{i} \geq 0, i=1,2, \ldots, 6,
$$

where $0 \leq q<1$, we see that $\psi$ is continuous and hence lower semicontinuous. Also (1.8) and (1.9) follow from (2.10) and (2.19) respectively.

Let $x_{0} \in X$ be arbitrary. Then as in Remark 1.1, the sequence $\left\langle r x_{n}\right\rangle_{n=1}^{\infty}$ with the choice (1.10) is a Cauchy sequence in $r(X)$ and hence converges in it. That is

$$
\lim _{n \rightarrow \infty} f x_{n-1}=\lim _{n \rightarrow \infty} r x_{n}=r p \text { for some } p \in X .
$$

It is not difficult to prove that $\lim _{n \rightarrow \infty} f x_{n}=r p$. In other words, $f$ and $r$ satisfy the $C L R_{r}$-property. Therefore it follows that $u=r p$ is a coindence point. From the proof given in [4] it follows that $u$ is a common fixed point of $f$ and $r$, wherein the symmetry of $X$ is not needed. Thus Corollary 2.4 is a significant generalization of Theorem 1.2.

\section{References}

[1] Z. Mustafa, B. Sims, A new approach to generalized metric spaces, Journal of Nonlinear and Convex Anal., 7, No. 2 (2006), 289-297.

[2] Z. Mustafa, H. Obiedat, F. Awawdeh, Some fixed point theorem for mapping on complete G-metric spaces, Fixed Point Theory and Applications (2008), Article ID 189870, 1-12.

[3] A. Rani, S. Kumar, N. Kumar, S.K. Garg, Common fixed point theorems for compatible and weakly compatible maps in G-materic spaces, Appl. Math. 3 (2012), 1128-1134.

[4] M. Abbas, B.E. Rhoades, Common fixed point results for noncommuting mappings without continuity in generalized metric spaces, Appl. Math. and Comp., 21, No. 5 (2009), 262-269.

[5] D. Surekha, T. Phaneendra, A generalized common fixed point theorem under an implicit relation, Demon. Math., 48, No-s: 2,3 (2015).

[6] V. Popa, Some fixed point theorems for compatible mappings satisfying an implicit relation, Demon. Math. 32 (1999), 157-163.

[7] Abdelkrim Aliouche, Common fixed point theorems via an implicit relation and new properties, Sochow Jour. Math., 33, No. 4 (2007), 593-601.

[8] Mohammad Akkouchi, Valeriu Popa, Well-posedness of a common fixed point problem for three mappings under strict contractive conditions, Buletin. Univers. Petrol-Gaze din Ploiesti, Seria Math. Inform. Fiz. 61 (2009), 1-10. 
[9] Abdelkrim Aliouche, Common fixed point theorems via implicit relations, Misc. Math. Notes, 11, No. 1 (2010), 3-12.

[10] V. Popa, A.M. Patriciu, Two general fixed point theorems for pairs of weakly compatible mappings in G-metric spaces, Novi Sad J. Math., 42, No. 2 (2012), 49-60. 